\title{
Updates on fits to electroweak parameters
}

\section{Jorge de Blas}

INFN, Sezione di Roma, Piazzale A. Moro 2, I-00185 Rome, Italy

E-mail: jorge.deblasmateo@roma1.infn.it

Marco Ciuchini*

INFN, Sezione di Roma Tre, Via della Vasca Navale 84, I-00146 Rome, Italy

E-mail: marco.ciuchinieroma3.infn.it

\section{Enrico Franco}

INFN, Sezione di Roma, Piazzale A. Moro 2, I-00185 Rome, Italy

E-mail: enrico.francolromal.infn.it

\section{Diptimoy Ghosh}

Department of Particle Physics and Astrophysics, Weizmann Institute of Science, Rehovot 76100, Israel

E-mail: diptimoy.ghosheweizmann.ac.il

\section{Satoshi Mishima}

Institute of Particle and Nuclear Studies, KEK, Tsukuba 305-0801, Japan

E-mail: satoshi.mishima@kek.jp

\section{Maurizio Pierini}

CERN, Geneva, Switzerland

E-mail: maurizio.pieriniecern.ch

\section{Laura Reina}

Physics Department, Florida State University, Tallahassee, FL 32306-4350, USA

E-mail: reina@hep. fsu.edu

\section{Luca Silvestrini}

INFN, Sezione di Roma, Piazzale A. Moro 2, I-00185 Rome, Italy

E-mail: luca.silvestrinieromal.infn.it

We review the status of global fits to electroweak precision observables and Higgs boson signal strenghts. Model-independent bounds on new physics contributions are derived in several setups: non-standard oblique corrections, modified $Z b \bar{b}, H V V$ and $H f \bar{f}$ couplings as well as generic contributions of dimension-six operators to the Standard Model Lagrangian. The constraining power of electroweak precision observables and Higgs boson signal strenghts is discussed.

XXVII International Symposium on Lepton Photon Interactions at High Energies

17-22 August 2015

Ljubljana, Slovenia

${ }^{*}$ Speaker. 


\section{Introduction}

Electroweak precision observables (EWPO) are a powerful tool for indirect searches of new physics (NP) beyond the Standard Model (SM). Indeed electroweak (EW) precision fits were able to anticipate the top and the Higgs masses before they were discovered. Now that the SM particle mass spectrum is complete, we can fully exploit the potential of EW precision fits in looking for NP.

Besides the EWPO, the discovery of the Higgs boson $H$ brings into the game new observables related to the Higgs boson couplings to vector bosons and fermions. Indeed the study of the properties of the Higgs boson discovered at the LHC, in conjunction with improved fits to EWPO that take into account recent progress in theoretical calculations and experimental measurements, offers a very constrained framework to explore new physics effects in the EW sector.

In this Proceedings we present an update of our Bayesian global fit of the EWPO and the Higgs signal strengths [1-3] performed using the HEP fit package ${ }^{1}$.

In particular, we study NP contributions to EWPO in a model-independent way by using $S, T$, $U$ oblique parameters [4,5], epsilon parameters [6-8], and modified $Z b \bar{b}$ couplings. Moreover, we put bounds on the scale factors $\kappa_{V}$ and $\kappa_{f}$ multiplying the $H V V$ and $H f \bar{f}$ couplings to EW vector bosons $(V)$ and fermions $(f)$ respectively using both EWPO and Higgs signal strengths. Other recent EW precision fits can be found, e.g., in refs. [9-13].

Finally we consider the effective Langrangian approach where NP contributions come from higher-dimensional operators. We fit the Wilson coefficients of dimension-six operators from the EWPO and Higgs data and translate the results into lower bounds on the NP scale. The effective Lagrangian approach in this context has received a lot of attention in recent years and several studies can be found in the literature $[2,3,12,14-58]$.

\section{Standard-Model fit}

In table 1, we present the EW precision fit of the SM with five inputs $\left(M_{Z}, m_{t}, M_{H}, \alpha_{s}\left(M_{Z}^{2}\right)\right.$, $\left.\Delta \alpha_{\text {had }}^{(5)}\left(M_{Z}^{2}\right)\right)$ and fifteen EWPO as constraints. Details on the analysis can be found in refs. [1,2].

The costraining power of the EW fit is dictated by the experimental uncertainties, as theory uncertainties are expected to be subdominant at present [59]. Compared to our previous analysis [2], we updated the value of the Higgs mass $m_{H}=(125.09 \pm 0.24) \mathrm{GeV}$ [60], whose precise determination has however little impact on the EWPO.

The top-quark mass is one of the most important parameters entering the EW fits. The improvement expected from the LHC will push forward the NP sensitivity of the EW precision tests. Yet, the pole mass of the top quark reported by the hadron-collider experiments is subject to ambiguities due to the actual mass definition and other technical details of the Monte Carlo programs used in experimental analyses [61-63], as well as possible higher-twist effects in the observables from which the top mass is extracted. It is believed that the ambiguity is at the level of hundreds $\mathrm{MeV}$. This year, new determination of the top mass from ATLAS (172.99 $\pm 0.48 \pm 0.78 \mathrm{GeV})$ [64], CMS $(172.44 \pm 0.13 \pm 0.47 \mathrm{GeV})[65]$, and from the Tevatron experiments $(174.34 \pm 0.37 \pm 0.52$

\footnotetext{
${ }^{1}$ The developer version of the HEPfit package is available under the GNU General Public License (GPL) from https://github.com/silvest/HEPfit.
} 


\begin{tabular}{lcccc}
\hline & Measurement & Fit result & Fit prediction & Pull \\
\hline$G_{\mu}\left[\mathrm{GeV}^{-2}\right]$ & $1.1663787 \times 10^{-5}$ & - & - & - \\
$\alpha$ & $1 / 137.035999074$ & - & - & - \\
$\alpha_{s}\left(M_{Z}^{2}\right)$ & $0.1185 \pm 0.0005$ & $0.1185 \pm 0.0005$ & $0.1184 \pm 0.0028$ & -0.0 \\
$\Delta \alpha_{\mathrm{had}}^{(5)}\left(M_{Z}^{2}\right)$ & $0.02750 \pm 0.00033$ & $0.02741 \pm 0.00026$ & $0.02725 \pm 0.00042$ & -0.5 \\
$M_{Z}[\mathrm{GeV}]$ & $91.1875 \pm 0.0021$ & $91.1879 \pm 0.0020$ & $91.199 \pm 0.011$ & +1.0 \\
$m_{t}[\mathrm{GeV}]$ & $173.34 \pm 0.76$ & $173.6 \pm 0.7$ & $176.9 \pm 2.5$ & +1.3 \\
$m_{H}[\mathrm{GeV}]$ & $125.09 \pm 0.24$ & $125.09 \pm 0.24$ & $97.40 \pm 25.59$ & -0.9 \\
\hline$M_{W}[\mathrm{GeV}]$ & $80.385 \pm 0.015$ & $80.365 \pm 0.006$ & $80.361 \pm 0.007$ & -1.4 \\
$\Gamma_{W}[\mathrm{GeV}]$ & $2.085 \pm 0.042$ & $2.0890 \pm 0.0005$ & $2.0890 \pm 0.0005$ & +0.1 \\
$\Gamma_{Z}[\mathrm{GeV}]$ & $2.4952 \pm 0.0023$ & $2.4945 \pm 0.0004$ & $2.4945 \pm 0.0004$ & -0.3 \\
$\sigma_{h}^{0}[\mathrm{nb}]$ & $41.540 \pm 0.037$ & $41.488 \pm 0.003$ & $41.488 \pm 0.003$ & -1.4 \\
$\sin ^{2} \theta_{\mathrm{eff}}^{\text {lept }}\left(Q_{\mathrm{FB}}^{\mathrm{had}}\right)$ & $0.2324 \pm 0.0012$ & $0.23144 \pm 0.00009$ & $0.23144 \pm 0.00009$ & -0.8 \\
$P_{\tau}^{\mathrm{pol}}$ & $0.1465 \pm 0.0033$ & $0.1477 \pm 0.0007$ & $0.1477 \pm 0.0007$ & +0.4 \\
$\mathscr{A}_{\ell}(\mathrm{SLD})$ & $0.1513 \pm 0.0021$ & $0.1477 \pm 0.0007$ & $0.1472 \pm 0.0008$ & -1.9 \\
$\mathscr{A}_{c}$ & $0.670 \pm 0.027$ & $0.6682 \pm 0.0003$ & $0.6682 \pm 0.0003$ & -0.1 \\
$\mathscr{A}_{b}$ & $0.923 \pm 0.020$ & $0.93466 \pm 0.00006$ & $0.93466 \pm 0.00006$ & +0.6 \\
$A_{\mathrm{FB}}^{0, \ell}$ & $0.0171 \pm 0.0010$ & $0.0164 \pm 0.0002$ & $0.0163 \pm 0.0002$ & -0.8 \\
$A_{\mathrm{FB}}^{0, c}$ & $0.0707 \pm 0.0035$ & $0.0740 \pm 0.0004$ & $0.0740 \pm 0.0004$ & +0.9 \\
$A_{\mathrm{FB}}^{0, b}$ & $0.0992 \pm 0.0016$ & $0.1035 \pm 0.0005$ & $0.1039 \pm 0.0005$ & +2.8 \\
$R_{\ell}^{0}$ & $20.767 \pm 0.025$ & $20.752 \pm 0.003$ & $20.752 \pm 0.003$ & -0.6 \\
$R_{c}^{0}$ & $0.1721 \pm 0.0030$ & $0.17224 \pm 0.00001$ & $0.17224 \pm 0.00001$ & +0.0 \\
$R_{b}^{0}$ & $0.21629 \pm 0.00066$ & $0.21578 \pm 0.00003$ & $0.21578 \pm 0.00003$ & -0.8 \\
\hline
\end{tabular}

Table 1: Experimental value, SM fit result, prediction and pull for input parameters and EWPOs. Fit predictions are fitted values obtained without using the corresponding experimental information; pulls are the difference between fit predictions and measurements in units of standard deviations.

$\mathrm{GeV}$ ) [66] have been presented. However, no official combination is available yet and we decided against using a naïve combination as the precision reached is approaching the uncertainty introduced by the ambiguity in the mass definition, requiring careful control and deep understanding of the experimental analyses in order to produce a solid result. Therefore, in the fit presented here, we stick to last year LHC-Tevatron combination $m_{t}=173.34 \pm 0.27 \pm 0.71 \mathrm{GeV}$ [67].

The globlal results of the fit in table 1 displays a general agreement of the EWPO with the SM predictions. The only deviations worth mentioning are the long-standing ones found in $\mathscr{A}_{\ell}$ and $A_{\mathrm{FB}}^{0, b}$, which are off by $-1.9 \sigma$ and $+2.8 \sigma$ respectively.

\section{Non-standard oblique corrections}

In this section, we present results of the fit for the oblique parameters $S, T$, and $U$ introduced in ref. $[4,5]$. Those parameters account for NP effects in the vacuum-polarization amplitudes of the EW gauge bosons. When the EW symmetry is realized linearly, $U$ is expected to be smaller than the others. The EWPO considered here depend on the three combinations of the oblique parameters 


\begin{tabular}{c|c|rrr}
\hline & Fit result & \multicolumn{3}{|c}{ Correlation Matrix } \\
\hline$S$ & $0.08 \pm 0.10$ & 1.00 & & \\
$T$ & $0.10 \pm 0.12$ & 0.85 & 1.00 & \\
$U$ & $0.01 \pm 0.09$ & -0.48 & -0.79 & 1.00 \\
\hline
\end{tabular}

Table 2: Results of the fit for the oblique parameters.

\begin{tabular}{c|c|lc}
\hline & Fit result & \multicolumn{2}{|l}{ Correlation Matrix } \\
\hline$S$ & $0.08 \pm 0.09$ & 1.00 & \\
$T$ & $0.10 \pm 0.07$ & 0.87 & 1.00 \\
\hline
\end{tabular}

Table 3: Results of the fit for the oblique parameters taking $U=0$.
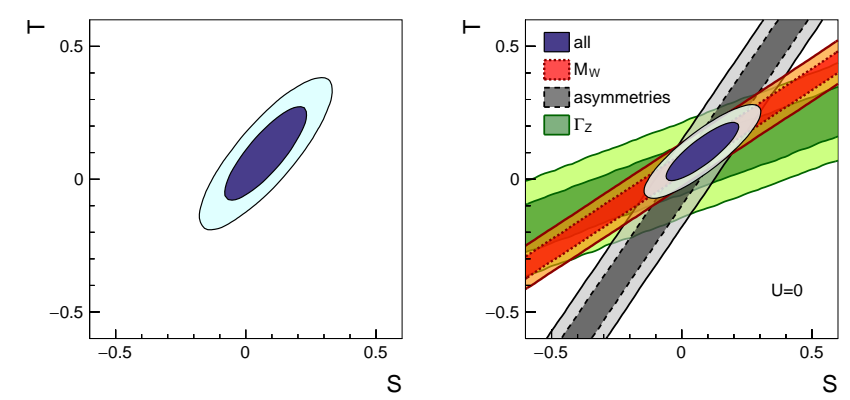

Figure 1: Two-dimensional probability distributions for the oblique parameters $S$ and $T$ varying $U$ (left) and for $U=0$ (right). The dark (light) region corresponds to 68\% (95\%) probability. In the right plot, the constraints from $M_{W}$, the asymmetry parameters $\sin ^{2} \theta_{\mathrm{eff}}^{\text {lept }}, P_{\tau}^{\mathrm{pol}}, A_{f}$ and $A_{\mathrm{FB}}^{0, f=\ell, c, b}$, and $\Gamma_{Z}$ are also shown.

introduced in ref. [1]. We summarize our results in tables 2 and 3 and in fig. 1. $S, T$, and $U$ are well compatible with zero: no evidence of NP in oblique corrections is found.

Next we consider the $\varepsilon_{i=1,2,3, b}$ parameters introduced in refs. [6-8]. Unlike the $S, T$, and $U$ parameters discussed above, the epsilon parameters involve SM contributions associated with the top quark and the Higgs boson, SM flavour non-universal vertex corrections, and further vacuumpolarization corrections [68]. Since the SM is now fully known and there is no longer need to disentangle top and Higgs contributions, we separate the genuine NP contribution from the SM one by introducing $\delta \varepsilon_{i}=\varepsilon_{i}-\varepsilon_{i, \mathrm{SM}}$ for $i=1,2,3, b$, where $\varepsilon_{i}$ are the original parameters and $\varepsilon_{i, \mathrm{SM}}$ contain the SM contribution only. The expressions of the EWPO in terms of $\delta \varepsilon_{i}$ can be found in ref. [2]. The results of our fit for the $\delta \varepsilon_{i}$ parameters are summarized in tables 4 and 5 , where $\delta \varepsilon_{2}$ and $\delta \varepsilon_{b}$ are set to be zero in the latter. The corresponding two-dimensional probability distributions for $\delta \varepsilon_{1}$ and $\delta \varepsilon_{3}$ are plotted in fig. 2. The results are consistent with the SM.

\section{Modified $Z b \bar{b}$ couplings}

We also consider the case where dominant NP contributions appear in the $Z b \bar{b}$ couplings. We

\begin{tabular}{l|r|rrrr}
\hline & \multicolumn{1}{|c|}{ Fit result } & \multicolumn{5}{|c}{ Correlation Matrix } \\
\hline$\delta \varepsilon_{1}$ & $0.0007 \pm 0.0010$ & 1.00 & & & \\
$\delta \varepsilon_{2}$ & $-0.0002 \pm 0.0009$ & 0.79 & 1.00 & & \\
$\delta \varepsilon_{3}$ & $0.0006 \pm 0.0009$ & 0.86 & 0.50 & 1.00 & \\
$\delta \varepsilon_{b}$ & $0.0003 \pm 0.0013$ & -0.32 & -0.31 & -0.21 & 1.00 \\
\hline
\end{tabular}

Table 4: Results of the fit for the $\delta \varepsilon_{i}$ parameters. 


\begin{tabular}{c|c|cc}
\hline & Fit result & \multicolumn{2}{|c}{ Correlations } \\
\hline$\delta \varepsilon_{1}$ & $0.0008 \pm 0.0006$ & 1.00 & \\
$\delta \varepsilon_{3}$ & $0.0007 \pm 0.0008$ & 0.87 & 1.00 \\
\hline
\end{tabular}

Table 5: Results of the fit for $\delta \varepsilon_{1}$ and $\delta \varepsilon_{3}$ taking $\delta \varepsilon_{2}=\delta \varepsilon_{b}=0$.
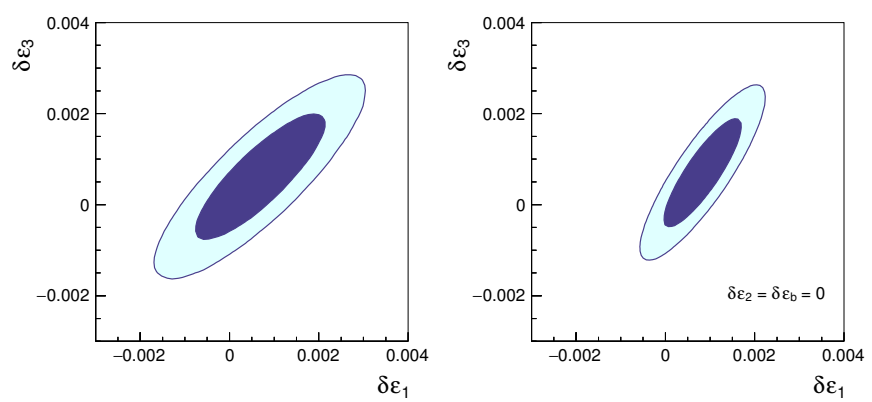

Figure 2: Two-dimensional probability distributions for $\delta \varepsilon_{1}$ and $\delta \varepsilon_{3}$ varying all $\delta \varepsilon_{i}$ parameters (left) or fixing $\delta \varepsilon_{2}=\delta \varepsilon_{b}=0$ (right). The dark (light) region corresponds to $68 \%$ (95\%) probability.

parameterize NP contributions to the $Z b \bar{b}$ couplings as follows:

$$
g_{i}^{b}=g_{i, \mathrm{SM}}^{b}+\delta g_{i}^{b} \quad \text { for } i=L, R \text { or } V, A,
$$

and we present results for both $V, A$ and $L, R$ couplings. Details on the definitions of these couplings can be found in ref. [1]. The EW precision fit finds four solutions for these couplings, but two of them are disfavored by the off-peak measurement of the forward-backward asymmetry in $e^{+} e^{-} \rightarrow$ $b \bar{b}$ [69]. In table 6 and fig. 3, we present only the solution closer to the SM. We observed deviations

\begin{tabular}{c|c|rc}
\hline & Fit result & \multicolumn{2}{|c}{ Correlations } \\
\hline$\delta g_{R}^{b}$ & $0.018 \pm 0.007$ & 1.00 & \\
$\delta g_{L}^{b}$ & $0.0029 \pm 0.0014$ & 0.90 & 1.00 \\
\hline$\delta g_{V}^{b}$ & $0.021 \pm 0.008$ & 1.00 & \\
$\delta g_{A}^{b}$ & $-0.015 \pm 0.006$ & -0.99 & 1.00 \\
\hline
\end{tabular}

Table 6: Results of the fit for the shifts in the $Z b \bar{b}$ couplings.
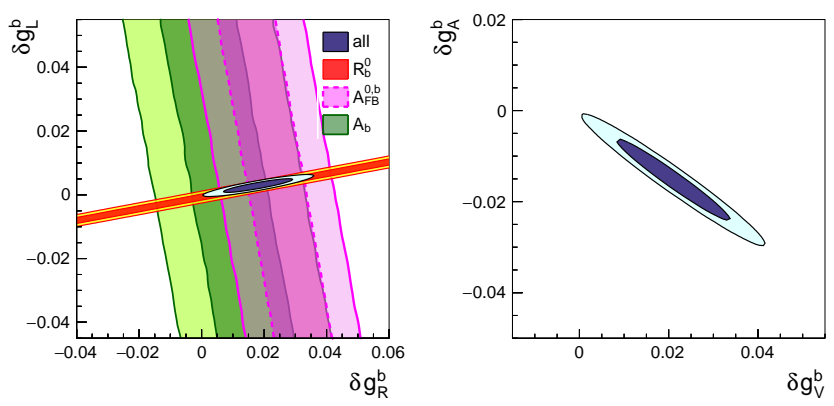

Figure 3: Two-dimensional probability distributions for $\delta g_{R}^{b}, \delta g_{L}^{b}$ (left), and $\delta g_{V}^{b}, \delta g_{A}^{b}$ (right). The dark (light) region corresponds to 68\% (95\%) probability. 
from zero of the parameters $\delta g_{i}^{b}$ reflect the deviation from the SM of the measured value of $A_{\mathrm{FB}}^{0, b}$.

\section{Modified Higgs couplings}

We consider a general effective Lagrangian for one light Higgs-like scalar field $H$, assuming an approximate custodial symmetry, flavour diagonal and universal corrections, and no other new light states below the cutoff scale [32,70-72]:

$$
\mathscr{L}_{\text {eff }}=\frac{v^{2}}{4} \operatorname{tr}\left(D_{\mu} \Sigma^{\dagger} D^{\mu} \Sigma\right)\left(1+2 \kappa_{V} \frac{H}{v}+\cdots\right)-m_{i} \bar{f}_{L}^{i}\left(1+2 \kappa_{f} \frac{H}{v}+\cdots\right) f_{R}^{i}+\cdots,
$$

where $v$ is the vacuum expectation value (VEV) of the Higgs field, and the longitudinal components of the $W$ and $Z$ bosons, $\chi^{a}(x)$, are described by the two-by-two matrix $\Sigma(x)=\exp \left(i \tau^{a} \chi^{a}(x) / v\right)$ with $\tau^{a}$ being the Pauli matrices. The deviations in the $H V V$ and $H f \bar{f}$ couplings are parameterized by the scale factors $\kappa_{V}$ and $\kappa_{f}$ respectively, which are equal to one in the SM.

\section{1 $H V V$ coupling only}

Let's first consider the EWPO and the scale factor $\kappa_{V}$ only. The oblique parameters $S$ and $T$ then receive the following contributions [73]:

$$
S=\frac{1}{12 \pi}\left(1-\kappa_{V}^{2}\right) \ln \left(\frac{\Lambda^{2}}{m_{H}^{2}}\right), \quad T=-\frac{3}{16 \pi c_{W}^{2}}\left(1-\kappa_{V}^{2}\right) \ln \left(\frac{\Lambda^{2}}{m_{H}^{2}}\right),
$$

where $\Lambda=4 \pi v / \sqrt{\left|1-\kappa_{V}^{2}\right|}$ is the cutoff scale of the effective Lagrangian. We present the results of the fit for $\kappa_{V}$ in table 7 and fig. 4 . The lower bound on $\kappa_{V}$ at $95 \%$ corresponds to a cutoff scale $\Lambda=13 \mathrm{TeV}$ if $\kappa_{V}$ is assumed to smaller than $1, \Lambda=8.5 \mathrm{TeV}$ if $\kappa_{V}$ is assumed to be larger than 1 , and $\Lambda=8.6 \mathrm{TeV}$ marginalizing over the sign of $1-\kappa_{V}{ }^{2}$ The fit disfavours values of $\kappa_{V}<1(9 \%$

\begin{tabular}{c|cc}
\hline & $68 \%$ & $95 \%$ \\
\hline$\kappa_{V}$ & $1.026 \pm 0.021$ & {$[0.987,1.071]$} \\
\hline
\end{tabular}

Table 7: Results of the fit for the scale factor $\kappa_{V}$ at $68 \%$ and $95 \%$ probabilities.
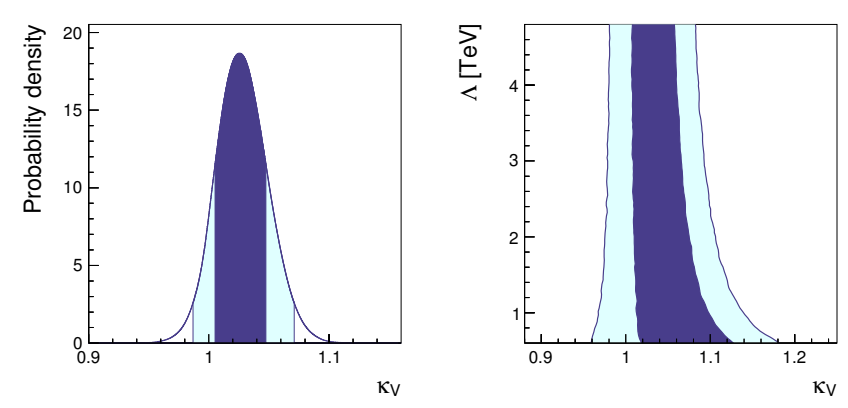

Figure 4: Left: probability distribution for $\kappa_{V}$. Right: two-dimensional probability distributions for $\kappa_{V}$ and $\Lambda$. The dark (light) region corresponds to $68 \%$ (95\%) probability.

\footnotetext{
${ }^{2}$ With respect to previous publications [1-3], here and in the EFT analysis of sec. 6, we adopt a new procedure to obtain the NP scale $\Lambda$ from the probability distributions of the NP couplings, detailed in ref. [74].
} 


\begin{tabular}{c|c|c|cc}
\hline & $68 \%$ & $95 \%$ & \multicolumn{2}{|c}{ Correlations } \\
\hline$\kappa_{V}$ & $1.01 \pm 0.04$ & {$[0.93,1.10]$} & 1.00 & \\
$\kappa_{f}$ & $1.03 \pm 0.10$ & {$[0.83,1.23]$} & 0.31 & 1.00 \\
\hline
\end{tabular}

Table 8: SM-like solution in the fit of $\kappa_{V}$ and $\kappa_{f}$ to the Higgs signal strengths.
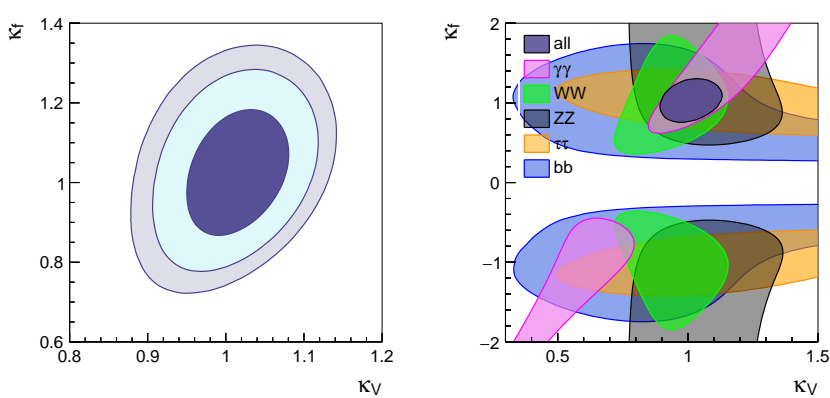

Figure 5: Left: two-dimensional probability distributions for $\kappa_{V}$ and $\kappa_{f}$ from the fit to the Higgs signal strengths. Contours correspond to $68 \%, 95 \%$, and $99 \%$ probability. Right: constraints from individual channels at $95 \%$ probability.

probability), expected for example in composite Higgs models. This problem can be alleviated by adding extra contributions to the oblique parameters [75-78]. In the right plot of fig. 4, we generalize the analysis allowing for $\Lambda<4 \pi v / \sqrt{\left|1-\kappa_{V}^{2}\right|}$, assuming that the oblique parameters are not affected by the dynamics at the cutoff. We find that $\kappa_{V}$ is constrained for $\Lambda>1 \mathrm{TeV}$.

\section{2 $H V V$ and $H f \bar{f}$ couplings}

Next we fit both scale factors $\kappa_{V}$ and $\kappa_{f}$ to the EWPO and the data for the Higgs signal strengths for $H \rightarrow \gamma \gamma$ [79,80], $H \rightarrow Z Z$ [81, 82], $H \rightarrow W^{+} W^{-}$[83-85], $H \rightarrow \tau^{+} \tau^{-}$[86, 87], and $H \rightarrow b \bar{b}$ [88-93]. We do not introduce couplings that are absent in the SM and we assume that there is no contribution from new particles in loop-induced couplings ( $H g g, H \gamma \gamma$, and $H Z \gamma)$. For the definition of the Higgs signal strengths and their relation with the scale factors, we refer the reader to ref. [94].

In table 8 we summarize the results of the fit for $\kappa_{V}$ and $\kappa_{f}$ from the Higgs signal strengths. In the left plot in fig. 5, we present two-dimensional probability distributions for $\kappa_{V}$ and $\kappa_{f}$. We plot only the region of positive $\kappa_{V}$, as the additional solutions are easily found considering that theoretical predictions are symmetric under the exchange $\left\{\kappa_{V}, \kappa_{f}\right\} \leftrightarrow\left\{-\kappa_{V},-\kappa_{f}\right\}$. The region with negative $\kappa_{f}$ is disfavored in the fit. The right plot in fig. 5 shows constraints from the individual decay channels.

We have also considered constraints from the EWPO with the formulae in eq. (5.2). Table 9

\begin{tabular}{c|c|c|cc}
\hline & $68 \%$ & $95 \%$ & \multicolumn{2}{|l}{ Correlations } \\
\hline$\kappa_{V}$ & $1.02 \pm 0.02$ & {$[0.99,1.06]$} & 1.00 & \\
$\kappa_{f}$ & $1.03 \pm 0.10$ & {$[0.85,1.23]$} & 0.15 & 1.00 \\
\hline
\end{tabular}

Table 9: Same as table 8 but considering both the Higgs signal strengths and the EWPO. 


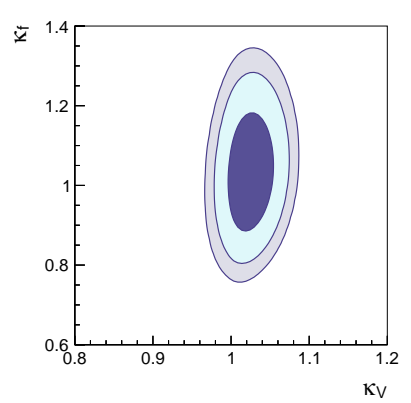

Figure 6: Two-dimensional probability distributions for $\kappa_{V}$ and $\kappa_{f}$ from the fit to the Higgs signal strengths and the EWPO. Contours correspond to $68 \%, 95 \%$, and $99 \%$ probability.

and fig. 6 show that the constraint on $\kappa_{V}$ from the EWPO is stronger than that from the Higgs signal strengths.

If we rescale the $H Z Z$ and $H W^{+} W^{-}$couplings independently splitting $\kappa_{V}$ into $\kappa_{Z}$ and $\kappa_{W}$ (keeping a unique $\kappa_{f}$ ), we obtain the results summarized in table 10 and the corresponding probability distributions shown in fig. 7, which are consistent with custodial symmetry. We have considered only the parameter space where both $\kappa_{W}$ and $\kappa_{Z}$ are positive as other solutions can be obtained considering that theoretical predictions are symmetric under the exchanges $\left\{\kappa_{W}, \kappa_{f}\right\} \leftrightarrow\left\{-\kappa_{W},-\kappa_{f}\right\}$ and/or $\kappa_{Z} \leftrightarrow-\kappa_{Z}$, where $\kappa_{Z}$ can flip the sign independent of $\kappa_{W}$ since the interference between the $W$ and $Z$ contributions to the vector-boson fusion cross section is negligible. Moreover, we do not fit to the EWPO, since setting $\kappa_{W} \neq \kappa_{Z}$ generates power divergences in the oblique corrections, making them sensitive to the UV completion of the theory.

Finally, we lift flavour universality and introduce different rescaling factors for charged leptons $\left(\kappa_{\ell}\right)$, up-type quarks $\left(\kappa_{u}\right)$, and down-type quarks $\left(\kappa_{d}\right)$, while keeping a unique rescaling factor $\kappa_{V}$ for both $H V V$ couplings. Again, we show only the parameter space where both $\kappa_{V}$ and $\kappa_{\ell}$ are positive as solutions in other regions can be obtained by applying the discrete symmetries $\kappa_{\ell} \leftrightarrow-\kappa_{\ell}$ and/or $\left\{\kappa_{V}, \kappa_{u}, \kappa_{d}\right\} \leftrightarrow\left\{-\kappa_{V},-\kappa_{u},-\kappa_{d}\right\}$. The constraints on the scale factors from the Higgs signal strengths are presented in table 11 and fig. 8. By adding the EWPO to the fit, the constraints

\begin{tabular}{c|c|c|rrr}
\hline & $68 \%$ & $95 \%$ & \multicolumn{3}{|c}{ Correlations } \\
\hline$\kappa_{W}$ & $1.00 \pm 0.05$ & {$[0.89,1.10]$} & 1.00 & & \\
$\kappa_{Z}$ & $1.07 \pm 0.11$ & {$[0.85,1.27]$} & -0.17 & 1.00 & \\
$\kappa_{f}$ & $1.01 \pm 0.11$ & {$[0.80,1.22]$} & 0.41 & -0.14 & 1.00 \\
\hline
\end{tabular}

Table 10: SM-like solution in the fit of $\kappa_{W}, \kappa_{Z}$, and $\kappa_{f}$ to the Higgs signal strengths.
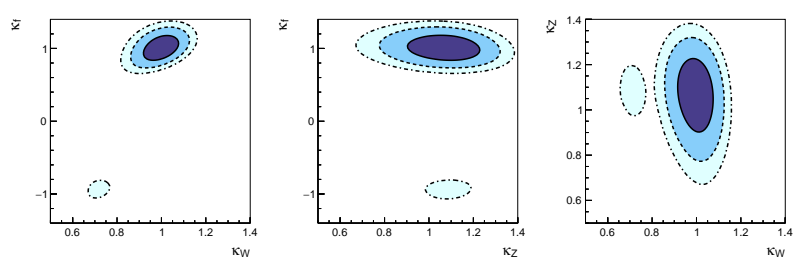

Figure 7: Two-dimensional probability distributions for $\kappa_{W}$ and $\kappa_{f}$ (left), $\kappa_{Z}$ and $\kappa_{f}$ (center), $\kappa_{W}$ and $\kappa_{Z}$ (right) from the fit to the Higgs signal strengths. Contours correspond to $68 \%, 95 \%$, and $99 \%$ probability. 


\begin{tabular}{c|c|c|cccc}
\hline & $68 \%$ & $95 \%$ & \multicolumn{4}{|c}{ Correlations } \\
\hline$\kappa_{V}$ & $0.97 \pm 0.08$ & {$[0.80,1.13]$} & 1.00 & & & \\
$\kappa_{\ell}$ & $1.01 \pm 0.14$ & {$[0.73,1.30]$} & 0.54 & 1.00 & & \\
$\kappa_{u}$ & $0.97 \pm 0.13$ & {$[0.73,1.25]$} & 0.43 & 0.41 & 1.00 & \\
$\kappa_{d}$ & $0.91 \pm 0.21$ & {$[0.48,1.34]$} & 0.81 & 0.61 & 0.77 & 1.00 \\
\hline
\end{tabular}

Table 11: SM-like solution in the fit of $\kappa_{V}, \kappa_{\ell}, \kappa_{u}$, and $\kappa_{d}$ to the Higgs signal strengths.
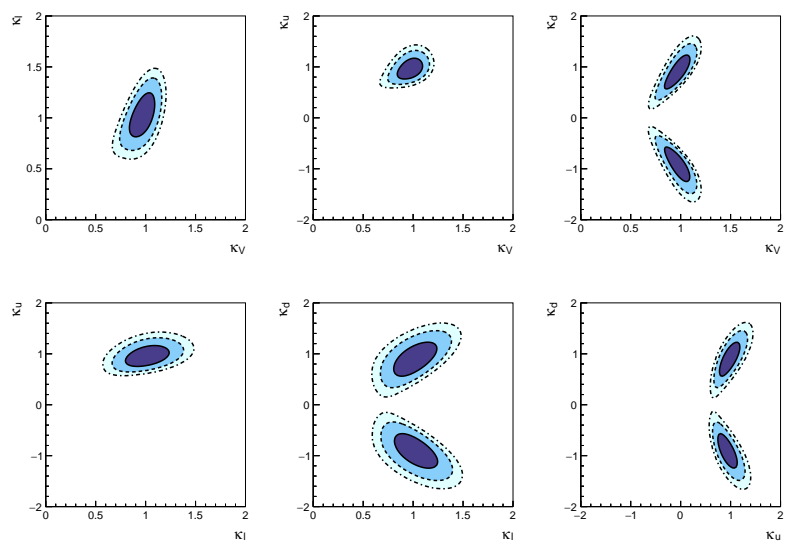

Figure 8: Two-dimensional probability distributions for $\kappa_{V}$ and $\kappa_{\ell}$ (top-left), $\kappa_{V}$ and $\kappa_{u}$ (top-center), $\kappa_{V}$ and $\kappa_{d}$ (top-right), $\kappa_{\ell}$ and $\kappa_{u}$ (bottom-left), $\kappa_{\ell}$ and $\kappa_{d}$ (bottom-center), $\kappa_{u}$ and $\kappa_{d}$ (bottom-right) from the fit to the Higgs signal strengths. Contours correspond to $68 \%, 95 \%$, and $99 \%$ probability (darker to lighter).

become stronger as shown in table 12 and fig. 9. No sign of violation of flavour universality is found in both cases.

\section{Constraints on dimension six effective operators}

In the effective field theory (EFT) approach, NP effects are described by higher-dimensional operators suppressed by the appropriate powers of the NP scale $\Lambda$. Considering the dimension-six operators, the effective Lagrangian can be written as

$$
\mathscr{L}_{\text {eff }}=\mathscr{L}_{\mathrm{SM}}+\frac{1}{\Lambda^{2}} \sum_{i} C_{i} \mathscr{O}_{i}+\ldots
$$

where $\mathscr{O}_{i}$ are the operators and $C_{i}$ are the corresponding Wilson coefficients that we want to constrain using the EWPO and Higgs data.

\begin{tabular}{c|c|c|cccc}
\hline & $68 \%$ & $95 \%$ & \multicolumn{4}{|c}{ Correlations } \\
\hline$\kappa_{V}$ & $1.02 \pm 0.02$ & {$[0.99,1.06]$} & 1.00 & & & \\
$\kappa_{\ell}$ & $1.07 \pm 0.12$ & {$[0.82,1.32]$} & 0.15 & 1.00 & & \\
$\kappa_{u}$ & $1.01 \pm 0.12$ & {$[0.80,1.27]$} & 0.10 & 0.24 & 1.00 & \\
$\kappa_{d}$ & $1.01 \pm 0.13$ & {$[0.77,1.31]$} & 0.31 & 0.38 & 0.78 & 1.00 \\
\hline
\end{tabular}

Table 12: Same as table 11 considering both the Higgs signal strengths and the EWPO. 

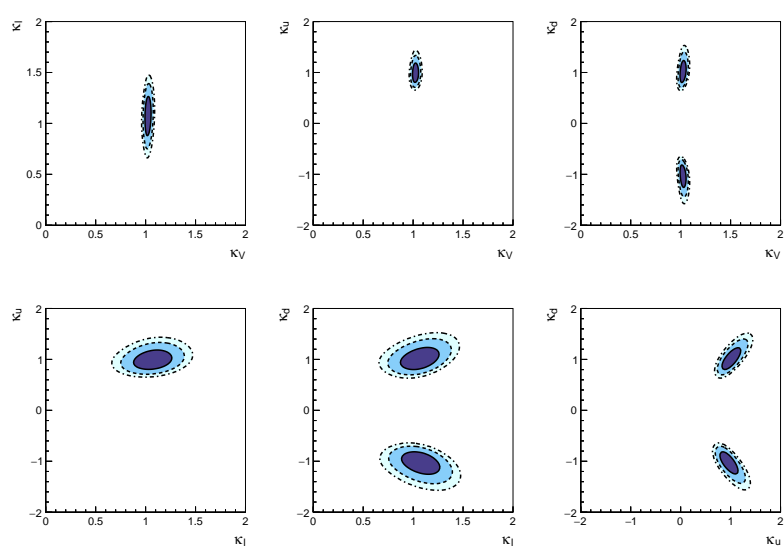

Figure 9: Same as fig. 8 considering both the Higgs signal strengths and the EWPO.

Starting from the operator basis defined in ref. [95] and considering only CP-even, flavourconserving and universal operators with at least one Higgs field which contribute to the observables considered here, one finds 16 operators:

$$
\begin{aligned}
\mathscr{O}_{H G} & =\left(H^{\dagger} H\right) G_{\mu v}^{A} G^{A \mu v}, & \mathscr{O}_{H W} & =\left(H^{\dagger} H\right) W_{\mu v}^{I} W^{I \mu v}, \\
\mathscr{O}_{H B} & =\left(H^{\dagger} H\right) B_{\mu v} B^{\mu v}, & \mathscr{O}_{H W B} & =\left(H^{\dagger} \tau^{I} H\right) W_{\mu v}^{I} B^{\mu \nu}, \\
\mathscr{O}_{H D} & =\left(H^{\dagger} D^{\mu} H\right)^{*}\left(H^{\dagger} D_{\mu} H\right), & \mathscr{O}_{H \square} & =\left(H^{\dagger} H\right) \square\left(H^{\dagger} H\right), \\
\mathscr{O}_{H L}^{(1)} & =\left(H^{\dagger} i \overleftrightarrow{D}{ }_{\mu} H\right)\left(\bar{L} \gamma^{\mu} L\right), & \mathscr{O}_{H L}^{(3)} & =\left(H^{\dagger} i \overleftrightarrow{D^{I}}{ }_{\mu} H\right)\left(\bar{L} \tau^{I} \gamma^{\mu} L\right), \\
\mathscr{O}_{H e} & =\left(H^{\dagger} i \overleftrightarrow{D}_{\mu} H\right)\left(\bar{e}_{R} \gamma^{\mu} e_{R}\right), & \mathscr{O}_{H Q}^{(1)} & =\left(H^{\dagger} i \overleftrightarrow{D}_{\mu} H\right)\left(\bar{Q} \gamma^{\mu} Q\right), \\
\mathscr{O}_{H Q}^{(3)} & =\left(H^{\dagger} i \overleftrightarrow{D}_{\mu}^{I} H\right)\left(\bar{Q} \tau^{I} \gamma^{\mu} Q\right), & \mathscr{O}_{H u} & =\left(H^{\dagger} i \overleftrightarrow{D}_{\mu} H\right)\left(\bar{u}_{R} \gamma^{\mu} u_{R}\right), \\
\mathscr{O}_{H d} & =\left(H^{\dagger} i \overleftrightarrow{D}_{\mu} H\right)\left(\bar{d}_{R} \gamma^{\mu} d_{R}\right), & \mathscr{O}_{e H} & =\left(H^{\dagger} H\right)\left(\bar{L} e_{R} H\right), \\
\mathscr{O}_{u H} & =\left(H^{\dagger} H\right)\left(\bar{Q} u_{R} \widetilde{H}\right), & \mathscr{O}_{d H} & =\left(H^{\dagger} H\right)\left(\bar{Q} d_{R} H\right),
\end{aligned}
$$

where $\tau^{I}$ are the three Pauli matrices and

$$
H^{\dagger} \overleftrightarrow{D}_{\mu} H=H^{\dagger}\left(D_{\mu} H\right)-\left(D_{\mu} H\right)^{\dagger} H, \quad H^{\dagger} \overleftrightarrow{D}_{\mu}^{I} H=H^{\dagger} \tau^{I}\left(D_{\mu} H\right)-\left(D_{\mu} H\right)^{\dagger} \tau^{I} H
$$

In addition, the four-fermion operator

$$
\mathscr{O}_{L L}=\left(\bar{L} \gamma_{\mu} L\right)\left(\bar{L} \gamma^{\mu} L\right)
$$

must be included in the analysis, as it can affect the extraction of the constant $G_{\mu}$ from the muon decay.

These operators introduce NP effects in the considered observables in various ways: purely bosonic operators change the $H W W, W W \gamma$, and $W W Z$ effective interaction vertices, thus contributing to the oblique parameters; operators with fermionic vector currents affect $H V f \bar{f}$ and $V f \bar{f}$ effective vertices; operators with fermionic scalar currents change the effective Yukawa couplings.

In our analysis, we consider only one Wilson coefficient at a time and fit it first to the EWPO and Higgs-boson observables separately, and then to the combination of both. Our results are summarized in table 13 where we show the $95 \%$ probability regions for the ratio $C_{i} / \Lambda^{2}$ in $\mathrm{TeV}^{-2}$. It 


\begin{tabular}{c|c|c|c} 
& Only EW & Only Higgs & EW + Higgs \\
\hline Coefficient & $\begin{array}{c}C_{i} / \Lambda^{2}\left[\mathrm{TeV}^{-2}\right] \\
\text { at } 95 \%\end{array}$ & $\begin{array}{c}C_{i} / \Lambda^{2}\left[\mathrm{TeV}^{-2}\right] \\
\text { at 95\% }\end{array}$ & $\begin{array}{c}C_{i} / \Lambda^{2}\left[\mathrm{TeV}^{-2}\right] \\
\text { at } 95 \%\end{array}$ \\
\hline$C_{H G}$ & -- & {$[-0.0047,0.0085]$} & {$[-0.0051,0.0087]$} \\
$C_{H W}$ & -- & {$[-0.034,0.016]$} & {$[-0.034,0.016]$} \\
$C_{H B}$ & -- & {$[-0.0082,0.0039]$} & {$[-0.0083,0.0036]$} \\
$C_{H W B}$ & {$[-0.0095,0.0033]$} & {$[-0.0085,0.018]$} & {$[-0.0069,0.0053]$} \\
$C_{H D}$ & {$[-0.031,0.0050]$} & {$[-1.4,1.4]$} & {$[-0.032,0.0058]$} \\
$C_{H \square}$ & -- & {$[-1.2,1.8]$} & {$[-1.2,1.8]$} \\
$C_{H L}^{(1)}$ & {$[-0.0055,0.012]$} & -- & {$[-0.005,0.012]$} \\
$C_{H L}^{(3)}$ & {$[-0.011,0.0055]$} & {$[-0.63,0.51]$} & {$[-0.011,0.0058]$} \\
$C_{H e}$ & {$[-0.017,0.0054]$} & -- & {$[-0.017,0.0059]$} \\
$C_{H Q}^{(1)}$ & {$[-0.028,0.042]$} & {$[-4.9,3.4]$} & {$[-0.028,0.043]$} \\
$C_{H Q}^{(3)}$ & {$[-0.011,0.013]$} & {$[-0.35,0.20]$} & {$[-0.012,0.013]$} \\
$C_{H u}$ & {$[-0.073,0.080]$} & {$[-3.3,2.7]$} & {$[-0.071,0.081]$} \\
$C_{H d}$ & {$[-0.14,0.068]$} & {$[-7.1,12]$} & {$[-0.14,0.068]$} \\
$\operatorname{Re} C_{e H}$ & -- & {$[-0.055,0.028]$} & {$[-0.055,0.027]$} \\
$\operatorname{Re} C_{u H}$ & -- & {$[-0.45,0.43]$} & {$[-0.44,0.44]$} \\
$\operatorname{Re} C_{d H}$ & -- & {$[-0.043,0.074]$} & {$[-0.042,0.072]$} \\
$C_{L L}$ & {$[-0.0096,0.023]$} & {$[-0.99,1.3]$} & {$[-0.0099,0.023]$} \\
\hline
\end{tabular}

Table 13: Results of the fit for the coefficients of the dimension six operators at $95 \%$ probability. The fit is performed switching on one operator at a time. Bounds from only EWPO, only Higgs signal strengths, and the two combined are shown separately.

can be observed that except for $\mathscr{O}_{H W B}$ the Electroweak precision constraints are much stronger than the Higgs signal strength data for all the operators which contribute to the EWPO. The strong constraint on $C_{H W B}$ from the Higgs data is due to its contribution to $H \rightarrow \gamma \gamma$ which is loop suppressed in the SM. The tight constraint on the operator $\mathscr{O}_{H G}$ can be understood in a similar way, as it contributes to the Higgs production through gluon fusion, which is also loop suppressed in the SM.

The bounds on the dimension-six operator coefficients in table 13 can also be translated into bounds on the NP scale for fixed values of coefficients. We show them in table 14 for three choices: $C_{i}=1, C_{i}=-1$, and $C_{i}= \pm 1$ obtained by marginalizing the probability distribution over the sign of the $C_{i}$. For $\left|C_{i}\right|=1$, our fit indicates that the scale of new physics $\Lambda$ is beyond LHC reach. However, for perturbative $C_{i}$, new physics at scales $\Lambda$ of $O(\mathrm{TeV})$ cannot be excluded.

Notice that, by switching on one operator at a time, we discarded the possibility of cancellations among different operators, which could weaken the constraints found on $\Lambda$. Yet, in EFTs, Wilson coefficients are independent couplings so that cancellations require introducing some fine tuning. This argument, however, is only partially satisfactory. In NP searches, we use EFTs to identify a pattern of correlations among Wilson coefficients which could point to a specific UV completion of the theory. To partially address this issue, we present the pictorial representation in fig. 10 of the correlations among coefficients and observables. In this plot, a circle indicates that a 


\begin{tabular}{c|ccc|ccc|ccc} 
& \multicolumn{3}{|c|}{ Only EW } & \multicolumn{3}{c|}{ Only Higgs } & \multicolumn{3}{c}{ EW + Higgs } \\
\hline & \multicolumn{3}{|c|}{$\Lambda[\mathrm{TeV}]$} & & \multicolumn{3}{c|}{$\Lambda[\mathrm{TeV}]$} & & \multicolumn{3}{c}{$\Lambda[\mathrm{TeV}]$} \\
$C_{i}$ & $C_{i}=1$ & $C_{i}=-1$ & $C_{i}= \pm 1$ & $C_{i}=1$ & $C_{i}=-1$ & $C_{i}= \pm 1$ & $C_{i}=1$ & $C_{i}=-1$ & $C_{i}= \pm 1$ \\
\hline$C_{H G}$ & -- & -- & -- & $11(68 \%)$ & $14(32 \%)$ & 12 & $11(69 \%)$ & $14(31 \%)$ & 12 \\
$C_{H W}$ & -- & -- & -- & $7.4(22 \%)$ & $5.8(78 \%)$ & 5.9 & $7.5(23 \%)$ & $5.8(77 \%)$ & 5.9 \\
$C_{H B}$ & -- & -- & -- & $16(22 \%)$ & $11(78 \%)$ & 12 & $16(24 \%)$ & $12(76 \%)$ & 12 \\
$C_{H W B}$ & $16(20 \%)$ & $11(80 \%)$ & 11 & $8.0(77 \%)$ & $10(23 \%)$ & 8.2 & $14(37 \%)$ & $12(63 \%)$ & 13 \\
$C_{H D}$ & $12(7 \%)$ & $5.9(93 \%)$ & 6.1 & $0.9(49 \%)$ & $0.9(51 \%)$ & 0.9 & $11(7 \%)$ & $5.9(93 \%)$ & 5.9 \\
$C_{H \square}$ & -- & -- & -- & $0.8(65 \%)$ & $0.9(35 \%)$ & 0.8 & $0.8(64 \%)$ & $0.9(36 \%)$ & 0.8 \\
$C_{H L}^{(1)}$ & $9.7(79 \%)$ & $13(21 \%)$ & 9.9 & -- & -- & -- & $9.8(78 \%)$ & $13(22 \%)$ & 10 \\
$C_{H L}^{(3)}$ & $15(25 \%)$ & $9.7(75 \%)$ & 9.7 & $1.4(41 \%)$ & $1.3(59 \%)$ & 1.4 & $12(28 \%)$ & $10(72 \%)$ & 10 \\
$C_{H e}$ & $13(14 \%)$ & $8.1(86 \%)$ & 8.3 & -- & -- & -- & $11(15 \%)$ & $8.1(85 \%)$ & 8.2 \\
$C_{H Q}^{(1)}$ & $5.1(64 \%)$ & $6.0(36 \%)$ & 5.3 & $0.5(36 \%)$ & $0.5(64 \%)$ & 0.5 & $5.1(65 \%)$ & $5.8(35 \%)$ & 5.3 \\
$C_{H Q}^{(3)}$ & $8.9(55 \%)$ & $9.8(45 \%)$ & 9.2 & $2.2(29 \%)$ & $1.8(71 \%)$ & 1.9 & $9.1(55 \%)$ & $9.3(45 \%)$ & 9.2 \\
$C_{H u}$ & $3.7(52 \%)$ & $3.8(48 \%)$ & 3.7 & $0.6(40 \%)$ & $0.6(60 \%)$ & 0.6 & $3.7(53 \%)$ & $3.8(47 \%)$ & 3.7 \\
$C_{H d}$ & $3.6(24 \%)$ & $2.8(76 \%)$ & 2.9 & $0.3(68 \%)$ & $0.4(32 \%)$ & 0.3 & $3.6(22 \%)$ & $2.8(78 \%)$ & 2.9 \\
$\operatorname{Re} C_{e H}$ & -- & -- & -- & $5.8(26 \%)$ & $4.4(74 \%)$ & 4.6 & $5.9(25 \%)$ & $4.5(75 \%)$ & 4.6 \\
$\operatorname{Re} C_{u H}$ & -- & -- & -- & $1.7(57 \%)$ & $1.4(43 \%)$ & 1.6 & $1.7(56 \%)$ & $1.5(44 \%)$ & 1.6 \\
$\operatorname{Re} C_{d H}$ & -- & -- & -- & $4.1(73 \%)$ & $4.2(27 \%)$ & 4.1 & $4.1(73 \%)$ & $4.3(27 \%)$ & 4.1 \\
$C_{L L}$ & $7.0(77 \%)$ & $9.8(23 \%)$ & 7.3 & $0.9(58 \%)$ & $1.0(42 \%)$ & 0.9 & $7.1(78 \%)$ & $9.1(22 \%)$ & 7.3 \\
\hline
\end{tabular}

Table 14: Lower bounds on the NP scale in TeV obtained using only EWPO, only Higgs data, and the two combined. For each data set and each operator, three bounds are presented: assuming $C_{i}=1$, assuming $C_{i}=-1$, and marginalizing the distribution over the sign of $C_{i}$ (denoted by $C_{i}= \pm 1$ ). In the first two cases, we report, next to the bound between parentheses, the probability found by the fit for that sign choice.

given operator contributes to a specific physical observable and the size of the circle represents the size of the contribution. Thus, small circles correspond to poorly bounded operators, while larger circles to more constrained ones. Fig. 10 is useful to see at a glance which observables are more affected by a given operator and, conversely, which operators are likely responsible for a deviation in a given observable. By construction, the displayed correlations only come from the operator structure, i.e. are low-energy correlations. Nevertheless, to some extent they could also provide information on the UV correlations among Wilson coefficients, as only operators with comparable bounds (namely same size circles) could have significant destructive interference. Of course, the ideal solution to study correlations would be fitting all the 17 operators at the same time, yet this task goes beyond the scope of the present study. This problem is sometimes circumvented in the literature by fitting a small subset of operators. While this allows for a complete control of the correlations, the choice of the subset is hardly justified in a fully model-independent analysis.

\section{Conclusions}

We have updated the EW precision fits in the SM and beyond taking into account recent theoretical and experimental developments. The results of the SM fit are presented in table 1, 


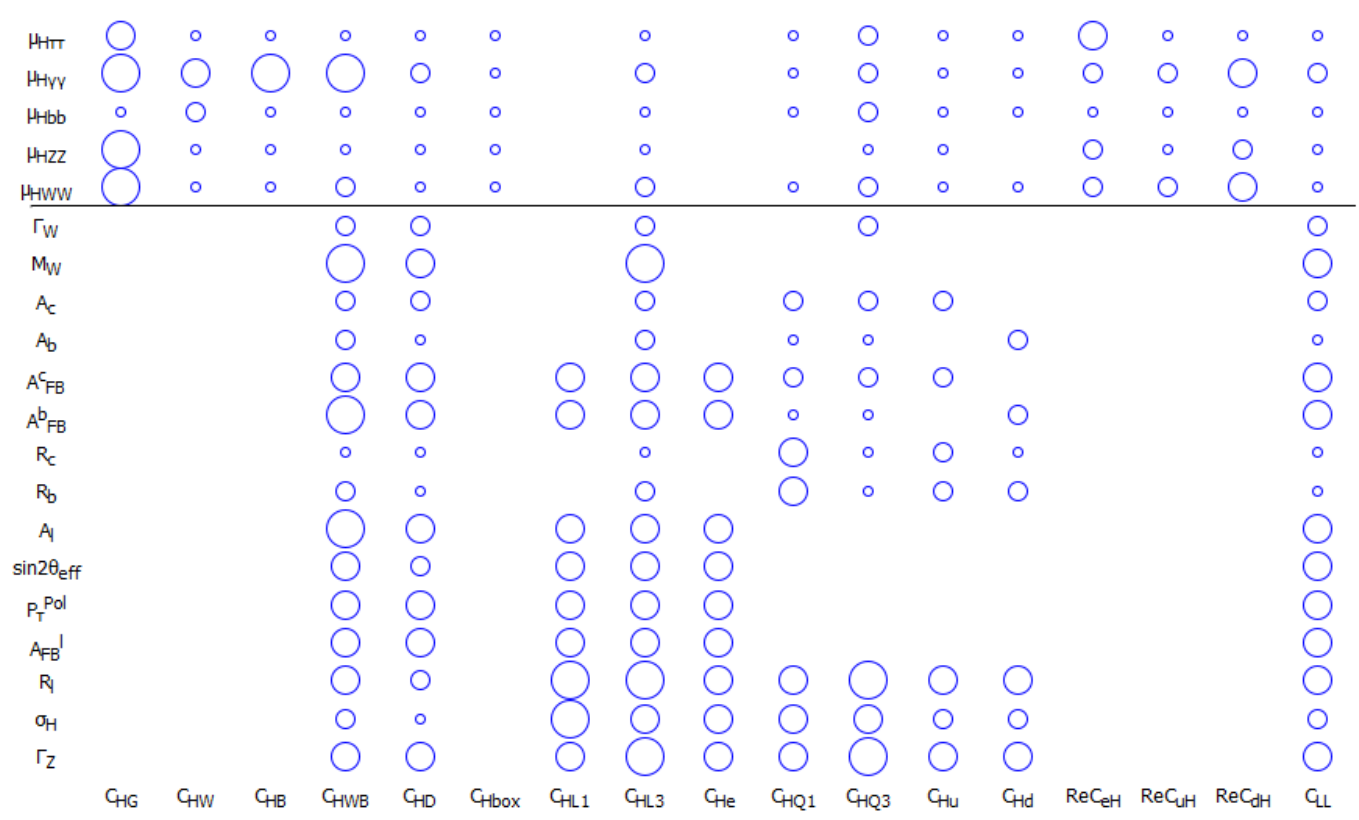

Figure 10: Correlation among Wilson coefficients and physical observables entering the precision fit. For each entry, the radius of the circle represents the constraint on the Wilson coefficient induced by that particular observable: the larger the circle, the more constrained the coefficient. There are five different increasing radii corresponding to upper bounds of $10^{-n}$ with $n=0,1,2,3,4$. No circle means no bound.

while the constraints on the NP parameters (the oblique and epsilon parameters, and the modified $Z b \bar{b}$ and $H V V$ couplings) are summarized in tables 2-7. Furthermore, we have performed fits of the scale factors of the Higgs couplings to the Higgs signal strengths and the EW precision data as summarized in tables 8-12. The same data have been used to constrain the Wilson coefficients of the dimension-six operators in the weak effective Lagrangian. Results are collected in tables 13 and 14. An extended discussion of these results and their implications will be presented in a future publication [74].

\section{Acknowledgments}

M.C. is associated to the Dipartimento di Matematica e Fisica, Università di Roma Tre, and E.F. and L.S. are associated to the Dipartimento di Fisica, Università di Roma "Sapienza". This research has received funding from the European Research Council under the European Union's Seventh Framework Programme (FP/2007-2013) / grants n. 267985 and n. 279972. The work of L.R. is supported in part by the U.S. Department of Energy under grant DE-FG02-13ER41942.

\section{References}

[1] M. Ciuchini, E. Franco, S. Mishima, and L. Silvestrini, Electroweak Precision Observables, New Physics and the Nature of a 126 GeV Higgs Boson, JHEP 08 (2013) 106, [1306 . 4644 ]. 
[2] M. Ciuchini, E. Franco, S. Mishima, M. Pierini, L. Reina, and L. Silvestrini, Update of the electroweak precision fit, interplay with Higgs-boson signal strengths and model-independent constraints on new physics, in International Conference on High Energy Physics 2014 (ICHEP 2014) Valencia, Spain, July 2-9, 2014, 2014. 1410.6940.

[3] J. de Blas, M. Ciuchini, E. Franco, D. Ghosh, S. Mishima, M. Pierini, L. Reina, and L. Silvestrini, Global Bayesian Analysis of the Higgs-boson Couplings, in International Conference on High Energy Physics 2014 (ICHEP 2014) Valencia, Spain, July 2-9, 2014, 2014. 1410.4204.

[4] M. E. Peskin and T. Takeuchi, A New constraint on a strongly interacting Higgs sector, Phys. Rev. Lett. 65 (1990) 964-967.

[5] M. E. Peskin and T. Takeuchi, Estimation of oblique electroweak corrections, Phys. Rev. D46 (1992) 381-409.

[6] G. Altarelli and R. Barbieri, Vacuum polarization effects of new physics on electroweak processes, Phys. Lett. B253 (1991) 161-167.

[7] G. Altarelli, R. Barbieri, and S. Jadach, Toward a model independent analysis of electroweak data, Nucl. Phys. B369 (1992) 3-32. [Erratum: Nucl. Phys.B376,444(1992)].

[8] G. Altarelli, R. Barbieri, and F. Caravaglios, Nonstandard analysis of electroweak precision data, Nucl. Phys. B405 (1993) 3-23.

[9] O. Eberhardt, G. Herbert, H. Lacker, A. Lenz, A. Menzel, U. Nierste, and M. Wiebusch, Impact of a Higgs boson at a mass of $126 \mathrm{GeV}$ on the standard model with three and four fermion generations, Phys. Rev. Lett. 109 (2012) 241802, [1209.1101].

[10] M. Baak, M. Goebel, J. Haller, A. Hoecker, D. Kennedy, R. Kogler, K. Moenig, M. Schott, and J. Stelzer, The Electroweak Fit of the Standard Model after the Discovery of a New Boson at the LHC, Eur. Phys. J. C72 (2012) 2205, [1209.2716].

[11] J. Erler, Tests of the Electroweak Standard Model, J. Phys. Conf. Ser. 485 (2014) 012010 , [1209.3324].

[12] J. de Blas, Electroweak limits on physics beyond the Standard Model, EPJ Web Conf. 60 (2013) 19008, [1307. 6173].

[13] Gfitter Group Collaboration, M. Baak, J. Cúth, J. Haller, A. Hoecker, R. Kogler, K. Mönig, M. Schott, and J. Stelzer, The global electroweak fit at NNLO and prospects for the LHC and ILC, Eur. Phys. J. C74 (2014) 3046, [1 407 . 3792].

[14] Z. Han and W. Skiba, Effective theory analysis of precision electroweak data, Phys. Rev. D71 (2005) 075009, [hep-ph/0 412166$].$

[15] F. del Aguila and J. de Blas, Electroweak constraints on new physics, Fortsch. Phys. 59 (2011) 1036-1040, [1105.6103].

[16] D. Carmi, A. Falkowski, E. Kuflik, T. Volansky, and J. Zupan, Higgs After the Discovery: A Status Report, JHEP 10 (2012) 196, [1207.1718].

[17] S. S. Biswal, R. M. Godbole, B. Mellado, and S. Raychaudhuri, Azimuthal Angle Probe of Anomalous HWW Couplings at a High Energy ep Collider, Phys. Rev. Lett. 109 (2012) 261801, [1203. 6285].

[18] S. Banerjee, S. Mukhopadhyay, and B. Mukhopadhyaya, New Higgs interactions and recent data from the LHC and the Tevatron, JHEP 10 (2012) 062, [1207 . 3588]. 
[19] T. Corbett, O. J. P. Eboli, J. Gonzalez-Fraile, and M. C. Gonzalez-Garcia, Constraining anomalous Higgs interactions, Phys. Rev. D86 (2012) 075013, [1207.1344].

[20] T. Corbett, O. J. P. Eboli, J. Gonzalez-Fraile, and M. C. Gonzalez-Garcia, Robust Determination of the Higgs Couplings: Power to the Data, Phys. Rev. D87 (2013) 015022, [1211. 4580].

[21] E. Massó and V. Sanz, Limits on anomalous couplings of the Higgs boson to electroweak gauge bosons from LEP and the LHC, Phys. Rev. D87 (2013), no. 3 033001, [1211.1320].

[22] D. Ghosh, R. Godbole, M. Guchait, K. Mohan, and D. Sengupta, Looking for an Invisible Higgs Signal at the LHC, Phys. Lett. B725 (2013) 344-351, [1211. 7015$].$

[23] B. Dumont, S. Fichet, and G. von Gersdorff, A Bayesian view of the Higgs sector with higher dimensional operators, JHEP 07 (2013) 065, [1304 . 3369].

[24] M. B. Einhorn and J. Wudka, The Bases of Effective Field Theories, Nucl. Phys. B876 (2013) 556-574, [1307.0478].

[25] M. B. Einhorn and J. Wudka, Higgs-Boson Couplings Beyond the Standard Model, Nucl. Phys. B877 (2013) 792-806, [1308.2255].

[26] E. Boos, V. Bunichev, M. Dubinin, and Y. Kurihara, Higgs boson signal at complete tree level in the SM extension by dimension-six operators, Phys. Rev. D89 (2014) 035001, [1309.5410].

[27] E. E. Jenkins, A. V. Manohar, and M. Trott, Renormalization Group Evolution of the Standard Model Dimension Six Operators I: Formalism and lambda Dependence, JHEP 10 (2013) 087, [1308.2627].

[28] E. E. Jenkins, A. V. Manohar, and M. Trott, Renormalization Group Evolution of the Standard Model Dimension Six Operators II: Yukawa Dependence, JHEP 01 (2014) 035, [1310 . 4838].

[29] R. Alonso, E. E. Jenkins, A. V. Manohar, and M. Trott, Renormalization Group Evolution of the Standard Model Dimension Six Operators III: Gauge Coupling Dependence and Phenomenology, JHEP 04 (2014) 159, [1312.2014].

[30] I. Brivio, T. Corbett, O. J. P. Éboli, M. B. Gavela, J. Gonzalez-Fraile, M. C. Gonzalez-Garcia, L. Merlo, and S. Rigolin, Disentangling a dynamical Higgs, JHEP 03 (2014) 024, [1311. 1823 ].

[31] C. Grojean, E. E. Jenkins, A. V. Manohar, and M. Trott, Renormalization Group Scaling of Higgs Operators and $\Gamma(h \rightarrow \gamma \gamma)$, JHEP 04 (2013) 016, [1301.2588].

[32] R. Contino, M. Ghezzi, C. Grojean, M. Muhlleitner, and M. Spira, Effective Lagrangian for a light Higgs-like scalar, JHEP 07 (2013) 035, [1303 . 3876].

[33] R. Contino, M. Ghezzi, C. Grojean, M. Mühlleitner, and M. Spira, eHDECAY: an Implementation of the Higgs Effective Lagrangian into HDECAY, Comput. Phys. Commun. 185 (2014) 3412-3423, [1403.3381].

[34] A. Pomarol and F. Riva, Towards the Ultimate SM Fit to Close in on Higgs Physics, JHEP 01 (2014) $151,[1308.2803]$.

[35] A. Alloul, B. Fuks, and V. Sanz, Phenomenology of the Higgs Effective Lagrangian via FEYNRULES, JHEP 04 (2014) 110, [1310.5150].+

[36] J. de Blas, M. Chala, and J. Santiago, Global Constraints on Lepton-Quark Contact Interactions, Phys. Rev. D88 (2013) 095011, [1307.5068].

[37] J. Elias-Miró, J. R. Espinosa, E. Masso, and A. Pomarol, Renormalization of dimension-six operators relevant for the Higgs decays $h \rightarrow \gamma \gamma, \gamma Z$, JHEP 08 (2013) 033, [1302 . 5661 ]. 
[38] J. Elias-Miro, J. R. Espinosa, E. Masso, and A. Pomarol, Higgs windows to new physics through d=6 operators: constraints and one-loop anomalous dimensions, JHEP 11 (2013) 066, [1308 1879 ].

[39] J. Elias-Miró, C. Grojean, R. S. Gupta, and D. Marzocca, Scaling and tuning of EW and Higgs observables, JHEP 05 (2014) 019, [1312. 2928].

[40] J. Ellis, V. Sanz, and T. You, Complete Higgs Sector Constraints on Dimension-6 Operators, JHEP 07 (2014) 036, [1404.3667].

[41] H. Belusca-Maito, Effective Higgs Lagrangian and Constraints on Higgs Couplings, 1404.5343.

[42] A. Biekötter, A. Knochel, M. Krämer, D. Liu, and F. Riva, Vices and virtues of Higgs effective field theories at large energy, Phys. Rev. D91 (2015) 055029, [1406.7320].

[43] M. Beneke, D. Boito, and Y.-M. Wang, Anomalous Higgs couplings in angular asymmetries of $H \rightarrow Z \ell^{+} \ell^{-}$and $e^{+} e^{-} \rightarrow H Z, J H E P 11$ (2014) 028, [1406.1361].

[44] M. Trott, On the consistent use of Constructed Observables, JHEP 02 (2015) 046, [1 409 . 7605 ].

[45] C. Hartmann and M. Trott, On one-loop corrections in the standard model effective field theory; the $\Gamma(h \rightarrow \gamma \gamma)$ case, JHEP 07 (2015) 151, [1505.02646].

[46] C. Hartmann and M. Trott, Higgs decay to two photons at one-loop in the SMEFT, 1507.03568.

[47] L. Berthier and M. Trott, Consistent constraints on the Standard Model Effective Field Theory, 1508.05060 .

[48] M. Ghezzi, R. Gomez-Ambrosio, G. Passarino, and S. Uccirati, NLO Higgs effective field theory and К-framework, JHEP 07 (2015) 175, [1505.0370 6].

[49] G. Buchalla and O. Cata, Effective Theory of a Dynamically Broken Electroweak Standard Model at NLO, JHEP 07 (2012) 101, [1203. 6510].

[50] G. Buchalla, O. Cata, and G. D'Ambrosio, Nonstandard Higgs couplings from angular distributions in $h \rightarrow Z \ell^{+} \ell^{-}$, Eur. Phys. J. C74 (2014), no. 3 2798, [1310.2574].

[51] G. Buchalla, O. Catá, and C. Krause, On the Power Counting in Effective Field Theories, Phys. Lett. B731 (2014) 80-86, [1312.5624].

[52] G. Buchalla, O. Cata, and C. Krause, A Systematic Approach to the SILH Lagrangian, Nucl. Phys. B894 (2015) 602-620, [1412.6356].

[53] G. Buchalla, O. Cata, A. Celis, and C. Krause, Note on Anomalous Higgs-Boson Couplings in Effective Field Theory, Phys. Lett. B750 (2015) 298-301, [1504.01707].

[54] C.-Y. Chen, S. Dawson, and C. Zhang, Electroweak Effective Operators and Higgs Physics, Phys. Rev. D89 (2014), no. 1 015016, [1311.3107].

[55] S. Dawson, I. M. Lewis, and M. Zeng, Effective field theory for Higgs boson plus jet production, Phys. Rev. D90 (2014), no. 9 093007, [1409.6299].

[56] D. Ghosh and M. Wiebusch, Dimension-six triple gluon operator in Higgs + jet observables, Phys. Rev. D91 (2015), no. 3 031701, [1411.2029].

[57] S. Dawson, I. M. Lewis, and M. Zeng, Usefulness of effective field theory for boosted Higgs production, Phys. Rev. D91 (2015) 074012, [1501.04103].

[58] J. de Blas, M. Chala, and J. Santiago, Renormalization Group Constraints on New Top Interactions from Electroweak Precision Data, JHEP 09 (2015) 189, [1 507 . 00757 ]. 
[59] A. Freitas, Electroweak precision tests in the LHC era and Z-decay form factors at two-loop level, in Proceedings, 12th DESY Workshop on Elementary Particle Physics: Loops and Legs in Quantum Field Theory (LL2014), 2014. 1406.6980.

[60] G. Aad et al. [ATLAS and CMS Collaborations], Phys. Rev. Lett. 114 (2015) 191803 doi:10.1103/PhysRevLett.114.191803 [arXiv:1503.07589 [hep-ex]].

[61] P. Z. Skands and D. Wicke, Non-perturbative QCD effects and the top mass at the Tevatron, Eur. Phys. J. C52 (2007) 133-140, [hep-ph/ 0703081$].$

[62] D. Wicke and P. Z. Skands, Non-perturbative QCD Effects and the Top Mass at the Tevatron, Nuovo Cim. B123 (2008) S1, [0 807 . 3248].

[63] A. Buckley et al., General-purpose event generators for LHC physics, Phys. Rept. 504 (2011) 145-233, [1101.2599].

[64] ATLAS Collaboration, G. Aad et al., Measurement of the top quark mass in the $t \bar{t} \rightarrow$ lepton + jets and $t \bar{t} \rightarrow$ dilepton channels using $\sqrt{s}=7 \mathrm{TeV}$ ATLAS data, Eur. Phys. J. C75 (2015), no. 7330 , [1503.05427].

[65] CMS Collaboration, V. Khachatryan et al., Measurement of the top quark mass using proton-proton data at $\sqrt{s}=7$ and $8 \mathrm{TeV}, 1509.04044$.

[66] CDF, D0 Collaboration, T. E. W. Group, Combination of CDF and DO results on the mass of the top quark using up to $9.7 \mathrm{fb}^{-1}$ at the Tevatron, 1407.2682.

[67] ATLAS, CDF, CMS, D0 Collaboration, First combination of Tevatron and LHC measurements of the top-quark mass, 1403.4427.

[68] R. Barbieri, A. Pomarol, R. Rattazzi, and A. Strumia, Electroweak symmetry breaking after LEP-1 and LEP-2, Nucl. Phys. B703 (2004) 127-146, [hep-ph / 0405040 ].

[69] D. Choudhury, T. M. P. Tait, and C. E. M. Wagner, Beautiful mirrors and precision electroweak data, Phys. Rev. D65 (2002) 053002, [hep-ph/ 0109097$].$

[70] G. F. Giudice, C. Grojean, A. Pomarol, and R. Rattazzi, The Strongly-Interacting Light Higgs, JHEP 06 (2007) 045, [hep-ph/ 0703164 ].

[71] R. Contino, C. Grojean, M. Moretti, F. Piccinini, and R. Rattazzi, Strong Double Higgs Production at the LHC, JHEP 05 (2010) 089, [1002 . 1011].

[72] A. Azatov, R. Contino, and J. Galloway, Model-Independent Bounds on a Light Higgs, JHEP 04 (2012) 127, [1202 . 3415]. [Erratum: JHEP04,140(2013)].

[73] R. Barbieri, B. Bellazzini, V. S. Rychkov, and A. Varagnolo, The Higgs boson from an extended symmetry, Phys. Rev. D76 (2007) 115008, [0 706.0432 ].

[74] J. de Blas, M. Ciuchini, E. Franco, D. Ghosh, S. Mishima, M. Pierini, L. Reina, and L. Silvestrini, in preparation.

[75] C. Grojean, W. Skiba, and J. Terning, Disguising the oblique parameters, Phys. Rev. D73 (2006) 075008, [hep-ph/0602154].

[76] A. Azatov, R. Contino, A. Di Iura, and J. Galloway, New Prospects for Higgs Compositeness in $h \rightarrow Z \gamma$, Phys. Rev. D88 (2013), no. 7 075019, [1308.2676].

[77] A. Pich, I. Rosell, and J. J. Sanz-Cillero, Viability of strongly-coupled scenarios with a light Higgs-like boson, Phys. Rev. Lett. 110 (2013) 181801, [1212 . 6769]. 
[78] A. Pich, I. Rosell, and J. J. Sanz-Cillero, Oblique S and T Constraints on Electroweak Strongly-Coupled Models with a Light Higgs, JHEP 01 (2014) 157, [1310 . 3121].

[79] CMS Collaboration, V. Khachatryan et al., Observation of the diphoton decay of the Higgs boson and measurement of its properties, Eur. Phys. J. C74 (2014), no. 10 3076, [14 07.0558 ].

[80] ATLAS Collaboration, G. Aad et al., Measurement of Higgs boson production in the diphoton decay channel in pp collisions at center-of-mass energies of 7 and $8 \mathrm{TeV}$ with the ATLAS detector, Phys. Rev. D90 (2014), no. 11 112015, [1408 . 7084].

[81] ATLAS Collaboration, G. Aad et al., Measurements of Higgs boson production and couplings in the four-lepton channel in pp collisions at center-of-mass energies of 7 and $8 \mathrm{TeV}$ with the ATLAS detector, Phys. Rev. D91 (2015), no. 1 012006, [1408.5191].

[82] CMS Collaboration, V. Khachatryan et al., Precise determination of the mass of the Higgs boson and tests of compatibility of its couplings with the standard model predictions using proton collisions at 7 and 8 TeV, Eur. Phys. J. C75 (2015), no. 5 212, [1412.8662].

[83] CMS Collaboration, S. Chatrchyan et al., Measurement of Higgs boson production and properties in the WW decay channel with leptonic final states, JHEP 01 (2014) 096, [1312 . 1129].

[84] ATLAS Collaboration, G. Aad et al., Observation and measurement of Higgs boson decays to WW* with the ATLAS detector, Phys. Rev. D92 (2015), no. 1 012006, [1412.2641].

[85] ATLAS Collaboration, G. Aad et al., Study of (W/Z)H production and Higgs boson couplings using $H \rightarrow W W^{*}$ decays with the ATLAS detector, JHEP 08 (2015) 137, [1506. 06641$]$.

[86] CMS Collaboration, S. Chatrchyan et al., Evidence for the $125 \mathrm{GeV} \mathrm{Higgs} \mathrm{boson} \mathrm{decaying} \mathrm{to} \mathrm{a} \mathrm{pair}$ of $\tau$ leptons, JHEP 05 (2014) 104, [1401. 5041 ].

[87] ATLAS Collaboration, G. Aad et al., Evidence for the Higgs-boson Yukawa coupling to tau leptons with the ATLAS detector, JHEP 04 (2015) 117, [1501. 04943 ].

[88] CDF Collaboration, T. Aaltonen et al., Combination fo Searches for the Higgs Boson Using the Full CDF Data Set, Phys. Rev. D88 (2013), no. 5 052013, [1301.6668].

[89] D0 Collaboration, V. M. Abazov et al., Combined search for the Higgs boson with the D0 experiment, Phys. Rev. D88 (2013), no. 5 052011, [1303.0823].

[90] CMS Collaboration, S. Chatrchyan et al., Search for the standard model Higgs boson produced in association with a W or a Z boson and decaying to bottom quarks, Phys. Rev. D89 (2014), no. 1 012003, [1310.3687].

[91] CMS Collaboration, V. Khachatryan et al., Search for the associated production of the Higgs boson with a top-quark pair, JHEP 09 (2014) 087, [1408 . 1682]. [Erratum: JHEP10,106(2014)].

[92] ATLAS Collaboration, G. Aad et al., Search for the $b \bar{b}$ decay of the Standard Model Higgs boson in associated $(W / Z) H$ production with the ATLAS detector, JHEP 01 (2015) 069, [1409.6212].

[93] ATLAS Collaboration, G. Aad et al., Search for the Standard Model Higgs boson produced in association with top quarks and decaying into b $\bar{b}$ in pp collisions at $\sqrt{s}=8 \mathrm{TeV}$ with the ATLAS detector, Eur. Phys. J. C75 (2015), no. 7 349, [1503.05066].

[94] LHC Higgs Cross Section Working Group Collaboration, J. R. Andersen et al., Handbook of LHC Higgs Cross Sections: 3. Higgs Properties, 1307.1347.

[95] B. Grzadkowski, M. Iskrzynski, M. Misiak, and J. Rosiek, Dimension-Six Terms in the Standard Model Lagrangian, JHEP 10 (2010) 085, [1 008 . 4884]. 